\title{
On the possible use of the MASURCA reactor as a flexible, high-intensity, fast neutron beam facility
}

\author{
Luca Dioni $^{1,2,3, \text { a }}$, Robert Jacqmin ${ }^{2}$, Marco Sumini $^{3}$, and Brian Stout ${ }^{1}$ \\ 1 Aix-Marseille University, Institut Fresnel, 13013 Marseille, France \\ 2 CEA Cadarache, DER/SPRC, 13108 Saint-Paul-lez-Durance, France \\ ${ }^{3}$ University of Bologna, Department of Industrial Engineering, 40136 Bologna, Italy
}

\begin{abstract}
In recent work [1,2], we have shown that the MASURCA research reactor could be used to deliver a fairly-intense continuous fast neutron beam to an experimental room located next to the reactor core. As a consequence of the MASURCA favorable characteristics and diverse material inventories, the neutron beam intensity and spectrum can be further tailored to meet the users' needs, which could be of interest for several applications. Monte Carlo simulations have been performed to characterize in detail the extracted neutron (and photon) beam entering the experimental room. These numerical simulations were done for two different bare cores: A uranium metallic core $\left(\sim 30 \%{ }^{235} \mathrm{U}\right.$ enriched $)$ and a plutonium oxide core $(\sim 25 \%$ Pu fraction, $\sim 78 \%$ ${ }^{239} \mathrm{Pu}$ ). The results show that the distinctive resonance energy structures of the two core leakage spectra are preserved at the channel exit. As the experimental room is large enough to house a dedicated set of neutron spectrometry instruments, we have investigated several candidate neutron spectrum measurement techniques, which could be implemented to guarantee well-defined, repeatable beam conditions to users. Our investigation also includes considerations regarding the gamma rays in the beams.
\end{abstract}

\section{Introduction}

MASURCA is a zero-power mock-up facility that has been mainly operated for getting experimental neutron physics validation data on sodium-cooled fast reactor cores. Thanks to the combination of several favourable features of the reactor building and of the core layouts, it is also possible to consider the development of a new capability: MASURCA as a beam facility.

In recent studies [1,2], we evaluated - by means of simulations - this possibility of operating MASURCA as a fast neutron source in which neutrons are "extracted" from the critical core using a radial channel (Fig. 1). In order to maximise the intensity and the fast-to-thermal neutron ratio of the beam at the end of the radial channel, entering the experimental zone, we looked into different core configurations. We concluded that MASURCA has the potential for delivering a continuous neutron beam with an intensity of $1.3 \times 10^{8}$ neutrons $\mathrm{cm}^{-2} \mathrm{~s}^{-1}$ (at $5 \mathrm{~kW}$ ) and with at least $89 \%$ of the neutrons having energies above $10 \mathrm{keV}$. We also concluded that these features, together with the characteristics of the reactor building, compare favourably with those of other reactorbased experimental facilities designed to deliver a fast neutron beam, such as YAYOI, TAPIRO and AFSR. This suggests that MASURCA could be used for a range of new applications: fast neutron shielding and transmission experiments, testing of advanced neutron detection systems, measurements of fast neutron cross sections, fast neutron radiography, etc.

\footnotetext{
a e-mail: luca.dioni@fresnel.fr; luca.dioni@cea.fr
}

In this study, in order to keep as many applications and options open, we shifted the core off-center by one meter, as much as practically feasible, towards the experimental zone for maximize the beam intensity and sensitivity to the core fissile constituents. The large and diverse inventories of materials available at MASURCA make it possible to represent a wide variety of cores, adaptable to the physicists' needs.

In the first part, for evaluating to what extent changes in the reactor core constituents affect the fast neutron beam, we present the comparison between two bare cores with a more "conventional" configuration of the MASURCA core $($ OffCIC $)$, already described in [2]. In the second part, we define spectrometric equipment that could be implemented in the MASURCA beam experimental configuration for neutron spectra measurement.

\section{The flexibility of MASURCA}

In MASURCA, experimental core configurations are constructed by loading subassemblies (called "tubes") filled with various types of materials (fissile, fertile, structure, coolant, absorber, air, etc.) in a square lattice. These materials are arranged in such a way as to represent average material concentrations and geometrical patterns of interest. The core, a group of tubes, can even be moved in the square lattice along the $X$ and $Y$ directions, the limitation is set by the position of the Safety Rods. There are 40 fixed positions in the lattice and as long as the core is constructed in a place where the SRs can be inserted, there is no regulatory preclusion.

In this work, we consider only shifted cores in the direction of the experimental zone, having dimensions that 


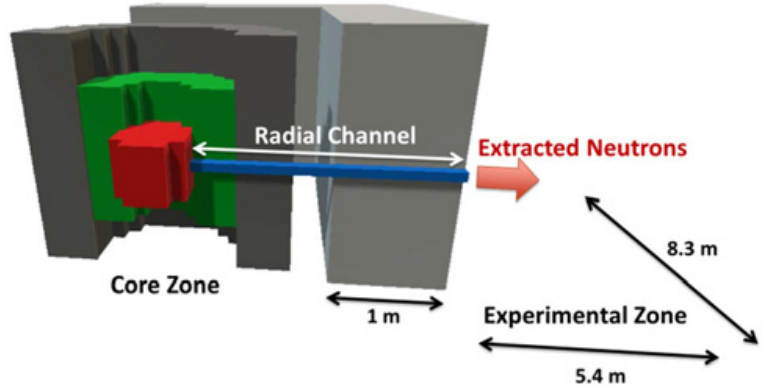

Figure 1. MASURCA Neutron Beam Configuration.

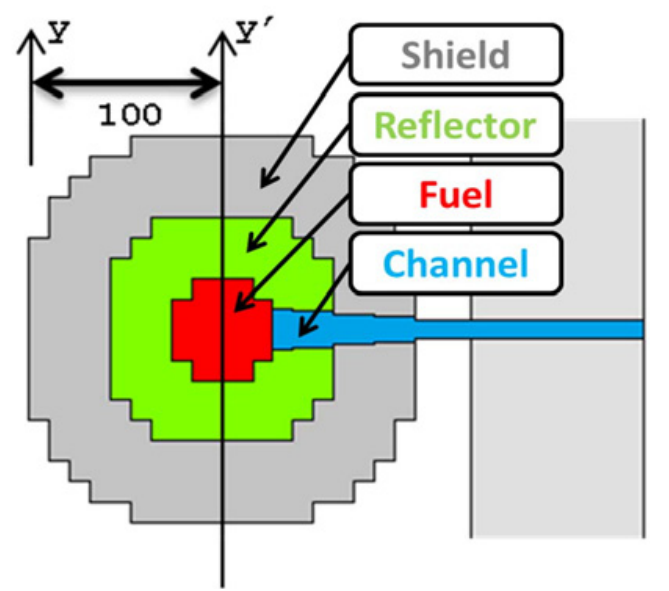

Figure 2. MASURCA in the OffCIC Core Configuration.

guarantee nearly critical configurations. All calculations were performed with the Monte Carlo code TRIPOLI-4 [3] and JEFF-3.1.1 nuclear data. The results are normalized to $5 \mathrm{~kW}$ total thermal power.

\subsection{The Off-Centered Inverted-Cone Model (OffCIC)}

The "OffCIC" experimental setup is characterized by a one meter shifted core in the $X-Y$ horizontal plane and by a $\sim 1.9 \mathrm{~m}$ long radial beam channel having an enlarged sectional area facing the fuel $\left(20.7 \times 20.7 \mathrm{~cm}^{2}\right)$, in order to maximize the fraction of neutrons coming from the core edge and entering the channel. The final part of the channel has a constant cross section of $10.6 \times 10.6 \mathrm{~cm}^{2}$ (Fig. 2).

All the material regions considered in the model are homogenous: the fuel region is made of metallic uranium fuel $\left(\sim 30 \%{ }^{235} \mathrm{U}\right.$ enriched) and sodium, the reflector region of stainless steel and sodium, and the shield region of stainless steel and absorbers. A concrete wall of about one meter separates the core from the experimental zone. The radial beam channel is filled with air.

\subsection{Bare core models}

We consider here two bare cores. The first one, named $U 30$, is a quasi-cylindrical core with a height of $60 \mathrm{~cm}$ and a radius of $22 \mathrm{~cm}$. In the core there is no sodium, only metallic fuel $\sim 30 \%{ }^{235} \mathrm{U}$ enriched. The second one, PITM, is characterized by the same height but with a radius of $24 \mathrm{~cm}$. The fuel is MOX, with a $\sim 25 \% \mathrm{Pu}$ fraction ( 78\% $\left.{ }^{239} \mathrm{Pu}\right)$.

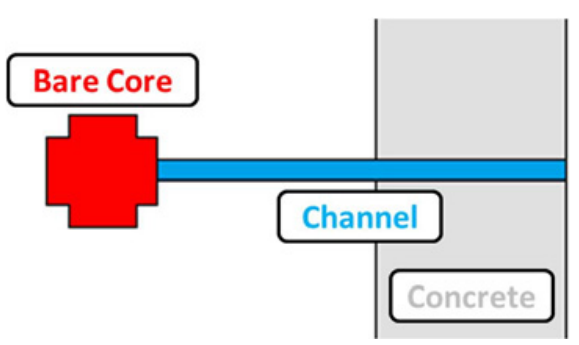

Figure 3. MASURCA Bare Core Configurations.

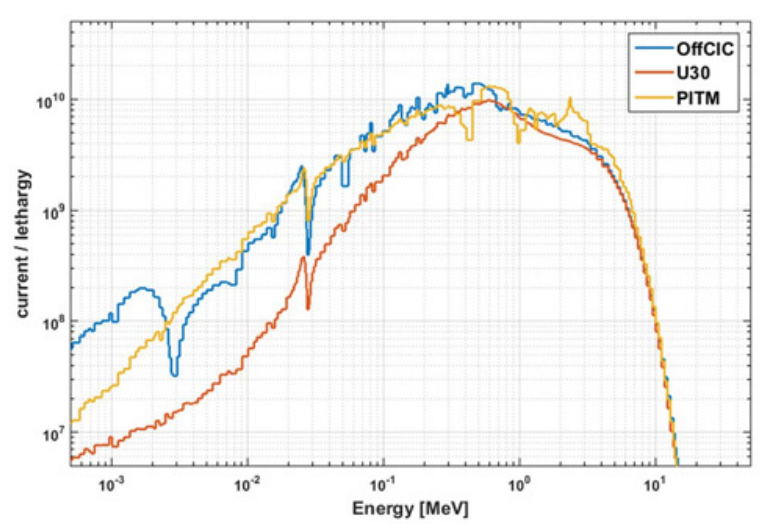

Figure 4. Neutron Spectra Entering the Radial Channel.

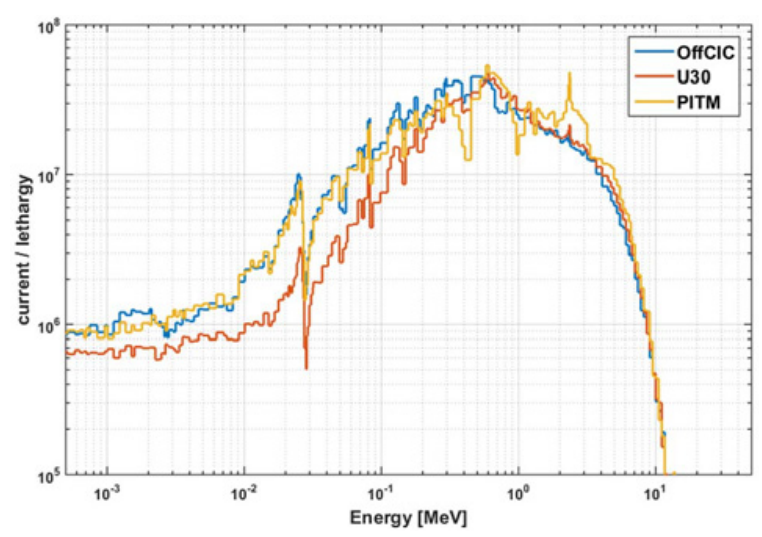

Figure 5. Neutron Spectra Exiting the Radial Channel.

The radial channel has a constant section of $10.6 \times$ $10.6 \mathrm{~cm}^{2}$ with a total length of about 2 meters. A schematic view of the models is reported in Fig. 3.

\subsection{Results}

The neutron outgoing currents for energies higher than $1 \mathrm{keV}$ at the beginning and at the end of the channel are reported in Figs. 4 and 5.

In the starting neutron spectra we can make several observations: i) the spectrum of $U 30$ is the hardest; ii) the spectrum of PITM inherits the resonance characteristics of ${ }^{16} \mathrm{O}$ - largely present in the MOX - at $435 \mathrm{keV}, 1$ and $2.35 \mathrm{MeV}$; iii) in the OffCIC spectrum, the presence of the ${ }^{23} \mathrm{Na}$ resonance at $2.8 \mathrm{keV}$ is clearly visible; and iv) all three spectra are signed by ${ }^{56} \mathrm{Fe}$ at $28 \mathrm{keV}$, the main structure material of the MASURCA tubes.

It is interesting to assess to what extent the distinctive features of the neutron spectra are maintained as neutrons travel into the radial channel, until they reach the experimental zone. 
Table 1. Total Neutron and Gamma Intensity at the End of the Channel.

\begin{tabular}{|c|c|c|c|c|}
\hline & $\begin{array}{c}\text { Total } \\
\text { Neutron } \\
\text { Intensity } \\
\left(\mathrm{n} \mathrm{cm}^{-2} \mathrm{~s}^{-1}\right)\end{array}$ & $\begin{array}{c}\text { Fast } \\
\text { Neutrons } \\
\text { Fraction } \\
(>10 \mathrm{keV})\end{array}$ & $\begin{array}{c}\text { Total } \\
\text { Gamma } \\
\text { Intensity } \\
\left(\gamma \mathrm{cm}^{-2} \mathrm{~s}^{-1}\right)\end{array}$ & $\mathrm{n}_{\text {fast }} / \gamma$ \\
\hline OffCIC & $1.32 \mathrm{E}+08$ & $89 \%$ & $2.52 \mathrm{E}+07$ & 4.66 \\
\hline U30 & $1.12 \mathrm{E}+08$ & $87 \%$ & $2.06 \mathrm{E}+07$ & 4.73 \\
\hline PITM & $1.35 \mathrm{E}+08$ & $86 \%$ & $2.68 \mathrm{E}+07$ & 4.33 \\
\hline
\end{tabular}

Starting from OffCIC we can clearly see that the ${ }^{23} \mathrm{Na}$ resonance "dip" is largely washed away. The neutrons interact strongly with the reflector and shield zones, and the resulting spectrum bears relatively little memory of the sodium resonance. Still, the characteristics over $10 \mathrm{keV}$ are well maintained.

Regarding U30 and PITM, we notice that the peculiarities of the two spectra are preserved. Notably, the hardness of the spectrum for $U 30$ and the oxygen fin energy structure for PITM, together with the iron one.

The general conclusion is that the neutron spectra at the end of the channel, entering the experimental zone, preserve much of the characteristics of the starting spectra above $10 \mathrm{keV}$. This finding suggests that an experimental characterization of the neutron beam should consider spectrometers capable of covering the energy domain over $10 \mathrm{keV}$.

\subsection{Gammas}

We analyse here the role and the weight of gamma rays at the end of the channel in relation to neutrons. A summary table of the neutron and gamma intensity (particles $\mathrm{cm}^{-2} \mathrm{~s}^{-1}$ ) is presented below (Table 1).

If we define the fast neutron fraction of interest as the neutrons with energy greater than $10 \mathrm{keV}$, we have a neutron-to-gamma ratio of 4 to 5 in that range. Preliminary simulations suggest that the introduction of filters, such as a few centimetres of lead, could raise this ratio by about one order of magnitude. An optimization is envisaged for the future for finding a good neutron-to-gamma ratio without perturbing too much the extracted neutron beam.

In terms of detection techniques, we are interested in neutron spectrometers insensitive to gammas or capable to discriminate well neutrons from gammas.

\section{Neutron beam characterization}

An important aspect of this work is to individuate and evaluate the relevant applicable technologies for the spectral characterization of the neutron beam. It is vital to comprehend which are the constraints imposed by the environment on the spectrometric system and, since the detection of neutrons strongly depends on their energy, the energy range of interest. We look for a measurement system covering the intermediate-to-fast neutron energy range, from $10 \mathrm{keV}$ to $10 \mathrm{MeV}$, in a radiation field that contains also many gammas and lower energy neutrons, here considered as unwanted background. As the reactor provides a continuous beam, techniques based on TOF are not directly applicable.

Comprehensive reviews and surveys of fast neutron spectroscopy methods can be found in the literature $[4,5]$.
Table 2. Detectors for Fast Neutron Spectroscopy.

\begin{tabular}{|c|c|c|c|}
\hline & $\begin{array}{c}\text { Discrim. } \\
\mathrm{n}-\gamma\end{array}$ & $\begin{array}{c}\text { Sensitivity } \\
\text { to thermal } \mathrm{n}\end{array}$ & $\begin{array}{c}\text { Energy } \\
\text { Domaine }\end{array}$ \\
\hline $\begin{array}{c}\text { Proportional } \\
\text { Counter }\end{array}$ & Good & No & $\begin{array}{c}10 \mathrm{keV}- \\
1 \mathrm{MeV}\end{array}$ \\
\hline $\begin{array}{c}\text { Organic } \\
\text { Scintillator }\end{array}$ & Average & No & $\begin{array}{c}>1 \mathrm{MeV} \\
(>400 \mathrm{keV}[6])\end{array}$ \\
\hline $\begin{array}{c}\text { p-recoil } \\
\text { Telescope }\end{array}$ & Good & No & $\begin{array}{c}>200 \mathrm{keV} \\
(>10 \mathrm{keV} \mathrm{[7]})\end{array}$ \\
\hline $\begin{array}{c}\text { Li-glass } \\
\text { Scintillator }\end{array}$ & Difficult & High & $\begin{array}{c}10 \mathrm{keV}- \\
1 \mathrm{MeV}\end{array}$ \\
\hline${ }^{3} \mathrm{He}$ & Good & High & $\begin{array}{c}10 \mathrm{MeV}- \\
1 \mathrm{MeV}\end{array}$ \\
\hline
\end{tabular}

Here we consider only detectors based on techniques in which the neutron is scattered and the energy of a recoiling nucleus is measured; and on the measurement of the energy released by charged particles in a neutroninduced nuclear reaction. Among them are ionization chambers, proportional counters, scintillators and proton recoil telescopes.

In Table 2, we summarized the properties of interest of several types of spectrometers, the focus is on neutrongamma discrimination and sensitivity to thermal neutrons.

${ }^{3} \mathrm{He}$ ionization chambers [4] are well-rounded, complete detectors for fast neutron spectroscopy. Nevertheless, the high sensitivity to thermal neutrons and the shortage of ${ }^{3} \mathrm{He}$ are expected to pose problems.

Li-glass inorganic scintillators [8] are characterized by a relatively fast response but with a poor light output. For these reasons they are utilized mainly in TOF spectroscopy. For $n-\gamma$ discrimination, a couple of scintillators should be used, one with only ${ }^{7} \mathrm{Li}$ (relatively inert to neutrons), the other enriched with ${ }^{6} \mathrm{Li}$.

Different types of proton recoil telescopes exist. In general, they are composed of a thin foil of hydrogenous material for $n-p$ conversion and then, at a selected angle, the charged particle detector. An interesting configuration is the one proposed in [9], where the protons are detected in a fixed-volume ionization chamber. The gas pressure is adjusted to the proton energy range and consequently to neutron energy. The drawback of this type of the detector is its low efficiency, usually one count per $10^{5}$ incident neutrons. Another interesting configuration is the one proposed in [7], where the energy detection lower limit is $10 \mathrm{keV}$.

Organic scintillators [10], both plastic and liquid, have the great advantage of being relatively cheap and easily adaptable in size and shapes. However, plastic scintillators are still not suitable for spectroscopy in mixed radiation field because the $n-\gamma$ discrimination capability is low. On the other hand, crystal scintillators like stilbene or anthracene - exhibit excellent $n-\gamma$ discrimination properties but suffer from a non-isotropic response. In [6], stilbene crystals are used for the neutron spectra characterization starting from $400 \mathrm{keV}$. Usually, liquid scintillators are preferred because they have the qualities of the two (plastic and crystal). The main drawback is the inflammability of the liquid, the container must be carefully prepared and the temperature conditions of the measurement under control.

Proportional Counters [11] that operated as recoil detectors can be of different geometries (spherical or 
cylindrical) and can be filled with different types of gasses (commonly used: $\mathrm{H}_{2}, \mathrm{CH}_{4}$ ). A set of proportional counters is typically used for covering a wide energy domain, like in [12], where $40 \mathrm{~mm}$ spherical hydrogen detectors with pressure of 100,400 and $1000 \mathrm{kPa}$ have been used for the measurement of an extracted neutron beam in the energy range $20-1200 \mathrm{keV}$.

For intermediate-to-fast neutron spectroscopy, a combination of detection techniques based on proportional counters, organic scintillators and proton recoil telescopes, is under study as it could provide the means for a finely energy characterization of the extracted neutron spectrum.

\section{Conclusion}

In this paper, we investigated to what extent changes in the MASURCA reactor core constituents affect the neutron beam that can be extracted thru an opening in the reactor side, and what detection techniques could be used for the characterization of the beams. We also evaluated the relative fraction of gamma rays.

Two bare cores were selected as "limit" cases for exacerbating the spectral properties of the fuel and we compared them with a more "conventional" configuration of the MASURCA core. The results show that over $10 \mathrm{keV}$ the extracted neutron spectra, at about two meters of distance, maintain well the initial spectral properties. In each configuration, a neutron-to-gamma ratio of 4-to-5 is found, without the use of any filter.

A brief survey of several fast neutron detection techniques established that a single detector can not be used for covering the energy domain from $10 \mathrm{keV}$ to $10 \mathrm{MeV}$, but that a possible solution could take the form of a multi-detector system based on proportional counters, organic (liquid or crystal) scintillators and proton recoil telescopes.

Such a beam plus detector system would be able to deliver a well-defined neutron spectrum and to guarantee its reproducibility, which could be of interest for various applications, e.g., for the calibration of detectors in the intermediate-to-fast energy region.

This work has been carried out thanks to the support of the A*MIDEX grant (No. ANR-11-IDEX-0001-02) funded by the French Government "Investissements d'Avenir" program.

\section{References}

[1] L. Dioni, R. Jacqmin, M. Sumini, B. Stout, ANIMMA 2015 (2015)

[2] L. Dioni, R. Jacqmin, M. Sumini, B. Stout, PHYSOR 2016 (to be published)

[3] J.-C. Trama et al., ICAPP 2011 (2011)

[4] G.F. Knoll, Radiation Detection and Measurement, Wiley and sons (2010)

[5] F.D. Brooks, H. Klein, NIM A 476, 1-11 (2002)

[6] V.A. Chernov, V.P. Semenov, L.A. Trykov, NIM A 476, 374-377 (2002)

[7] D. Maire et al., IEEE Transaction on Nuclear Science 63 (2016)

[8] A. Sardet, Thèse de doctorat (2015)

[9] P. Marini, L. Mathieu, M. Aïche, S. Czajkowski, B. Jurado, I. Tsekhanovich, WONDER 2015 (2016)

[10] H. Klein, Radiation Protection Dosimetry 107, 95-109 (2003)

[11] H. Tagziria, W. Hansen, Radiation Protection Dosimetry 107, 73-93 (2003)

[12] B. Jansky, E. Novak, NIM A 735, 390-398 (2014) 